\title{
Meta-ecosystem dynamics drive the spatial distribution of functional groups in river networks
}

\author{
Claire Jacquet ${ }^{1,2,3, *, \dagger}$, Luca Carraro ${ }^{1,2, *}$, Florian Altermatt ${ }^{1,2}$ \\ ${ }^{1}$ Department of Aquatic Ecology, Swiss Federal Institute of Aquatic Science and Technology, Eawag, Dübendorf, \\ Switzerland \\ ${ }^{2}$ Department of Evolutionary Biology and Environmental Studies, University of Zurich, Zürich, Switzerland \\ ${ }^{3}$ Complex Systems Lab, INRAE - Centre Clermont-Auvergne-Rhône-Alpes, Aubière, France \\ * These authors contributed equally to this work \\ + Corresponding author: claire.jacquet@inrae.fr
}

\section{Abstract}

2 Spatial flow of material and resources is a central process structuring ecological communities. The metaecosystem concept provides a theoretical framework to study the interplay between local and regional flows of resources and their implications for ecosystem dynamics and functioning. Yet, meta-ecosystem theory has been applied to highly simplified systems, and the effects of meta-ecosystem dynamics in real-world landscapes, characterized by specific spatial structures, remain largely unexplored. Here, we develop a spatially explicit meta-ecosystem model for dendritic river networks based on a highly realistic landscape matrix. By formalizing a seminal concept in freshwater ecology, we show that the spatial distributions and regional biomass of major functional groups observed in stream communities are determined by specific rates of resource flows. Overall, high rates of resource flow have a negative effect on the regional biomass of all the functional groups studied and can lead to extinctions at the meta-ecosystem scale.

Keywords: metaecosystem, dendritic network, riverine ecosystem, river continuum concept, trophic groups, macroinvertebrates, resource transportation, spatial flows, spatially explicit model. 


\section{INTRODUCTION}

Ecosystems are open to flows of materials, organisms, and energy, and understanding the effects of these flows on ecosystem structure and functioning is a central goal of ecological research [Polis et al., 2004]. By integrating the local production and movement of abiotic resources into metacommunity theory, the meta-ecosystem framework allows investigating feedback processes between community and resource dynamics across spatial scales [Gounand et al., 2018a; Loreau et al., 2003; Massol et al., 2011]. A fundamental prediction of meta-ecosystem theory is that the structure of an ecosystem in one locality depends on the rate of resource spatial flows and on the composition of the ecosystem(s) to which it is connected [Gravel et al., 2010a; Loreau et al., 2003; Massol et al., 2011].

Seminal work on meta-ecosystems was developed on simplified landscapes [Loreau et al., 2003; Loreau and Holt, 2004], often represented by two-patch ecosystems [Gravel et al., 2010a,b; Marleau et al., 2010]. This simple representation allowed an analytical investigation of the interplay between local and regional flows of matter and their implications for community dynamics [Gravel et al., 2010a; Loreau et al., 2003; Leroux and Loreau, 2012; Massol et al., 2011]. More recent studies focusing on larger spatial networks outlined the importance of spatial structure and movement rates of organisms and materials to promote meta-ecosystem stability [Marleau et al., 2014; Gravel et al., 2016]. However, one enduring limitation of current meta-ecosystem models is the abstract representation of the landscape [Gounand et al., 2018a], which is often described by random or Cartesian spatial networks [Marleau et al., 2014; Gravel et al., 2016].

In contrast, the physical structure of real-world landscapes constrains organisms' movement and resource flows and is likely to have broad-scale effects on the spatial distributions of resources and the organisms that feed upon them [Harvey et al., 2017a, 2020; Leroux and Loreau, 2008; Montagano et al., 2018; Schmitz et al., 2018]. Hence, the development of spatially explicit meta-ecosystem models is needed to understand the interactive effects of landscape structure and resource spatial dynamics on ecosystem structure and functioning [Massol et al., 2011]. In a similar way, the integration of spatially explicit (and then spatially realistic) landscapes was critical in advancing metapopulation and metacommunity ecology from a purely theoretical concept to a foundation for contemporary community ecology and conservation biology [Albert et al., 2013; Carrara et al., 2012; Hanski and Ovaskainen, 2000; Saccheri et al., 1998]. The application of meta-ecosystem models to real-world landscapes requires knowledge on the specific physical attributes of a landscape that constrain the direction and rates of resource flows among localities [Polis et al., 1997, 2004; Montagano et al., 2018]. However, this information remains scarce or ignored for most 
ecosystem types (but see the recent synthesis of Gounand et al. [2018b]).

In parallel to meta-ecosystem research, a large body of literature in freshwater ecology has outlined the critical influence of directional resource flows, both from the surrounding terrestrial environment and from upstream reaches, on the structure of local stream communities [Soininen et al., 2015; Abelho and Descals, 2019; Bartels et al., 2012]. In particular, the River Continuum Concept (RCC) [Vannote et al., 1980] proposes that commonly observed changes in the functional composition of freshwater invertebrates along a longitudinal river gradient reflect systematic shifts in types and locations of food resources with stream size. The RCC focuses on invertebrates that are large enough to be seen with the naked eye (also called macroinvertebrates), encompassing the most common organisms in riverine ecosystems [Wallace and Webster, 1996]. The RCC states that resource input, transport and use by macroinvertebrate functional groups are principally determined by the physical attributes of river networks (e.g., stream width, depth and water velocity).

The RCC is likely the most influencing conceptual framework in freshwater and stream ecology and is among the most commonly cited works in this field (totalling $>6000$ citations in the Scopus database), prompting empirical and theoretical works assessing its strengths and limitations (see Doretto et al. [2020] for a recent review). The parallel between meta-ecosystem theory and the mechanisms formulated in the RCC has been acknowledged in several recent studies [Massol et al., 2011; Doretto et al., 2020; Gounand et al., 2018a; Harvey et al., 2020]. However, a formal integration of the verbal arguments of the RCC within a meta-ecosystem model is still lacking.

In this study, we develop a spatially explicit meta-ecosystem model for generic river networks to address the effect of resource spatial dynamics on the functional composition of stream communities along a longitudinal river gradient, as postulated in the River Continuum Concept [Vannote et al., 1980]. We analyse different scenarios of meta-ecosystem dynamics and show that the broad-scale variations of functional composition observed in stream communities is determined by specific rates of resource flows. We find that increased rates of resource flows have a negative impact on the regional biomass of all the functional groups studied and can lead to extinctions at the meta-ecosystem scale. These findings suggest that human-induced modifications of meta-ecosystem dynamics in river networks can have a broad-scale impact on community functional composition and ecosystem functioning. 


\section{MATERIALS AND METHODS}

\section{Meta-ecosystem structure and dynamics}

We considered a riverine meta-ecosystem composed of a set of local ecosystems, each of them defined by a river reach and its surrounding terrestrial area, which are spatially connected via resource flow across the river network. Each local ecosystem is composed of four abiotic resources, a group of primary producers and five groups of consumers, corresponding to the groups and resource types defined in the River Continuum Concept and commonly used in freshwater ecology [Doretto et al., 2020; Larsen et al., 2019; Vannote et al., 1980; Wallace and Webster, 1996]. First, grazers $G$ feed on primary producers $P$ (algae or aquatic rooted vascular plants), which are supported by nutrients $N$ (e.g., nitrogen) and light (Fig. 1). Second, three independent functional groups feed on different forms of particulate organic matter derived from decomposed terrestrial leaf litter: shredders $S$, collectors $C$, and filterers $F$ feed on coarse (CPOM), fine (FPOM), and dissolved (DOM) particulate organic matter, respectively. Finally, predators $R$ feed on other groups of consumers, that is $G, S, C$ and $F$ (Fig. 1).

The abiotic resources present in a local ecosystem originate both from local terrestrial inputs of $N$, $C P O M$ and $D O M$ and from upstream reaches via hydrological transport of $N, C P O M, F P O M$ and DOM. Consequently, local ecosystems are connected through hydrological transport of resources, and the functional composition of upstream ecosystems determines the amount of resources available for the ecosystems situated downstream. We focused our analysis on an equilibrium state for the meta-ecosystem, and did not consider the temporal fluctuation of resource inputs, such as seasonality in resource availability or stream flow. We further hypothesized that living organisms only move within river reaches and do not disperse across local ecosystems. Our meta-ecosystem model is expressed by the following set of Lotka-Volterra ordinary differential equations:

$$
\begin{aligned}
\frac{\mathrm{d} P_{i}}{\mathrm{~d} t} & =\epsilon_{P} \alpha_{P} l_{i} P_{i} N_{i}-\alpha_{G} G_{i} P_{i}-\mu_{P} P_{i}-\beta_{P} P_{i}^{2} \\
\frac{\mathrm{d} G_{i}}{\mathrm{~d} t} & =\epsilon_{G} \alpha_{G} G_{i} P_{i}-\alpha_{R} R_{i} G_{i}-\mu_{G} G_{i}-\beta_{G} G_{i}^{2} \\
\frac{\mathrm{d} S_{i}}{\mathrm{~d} t} & =\epsilon_{S} \alpha_{S} S_{i} C P O M_{i}-\alpha_{R} R_{i} S_{i}-\mu_{S} S_{i}-\beta_{S} S_{i}^{2} \\
\frac{\mathrm{d} C_{i}}{\mathrm{~d} t} & =\epsilon_{C} \alpha_{C} C_{i} F P O M_{i}-\alpha_{R} R_{i} C_{i}-\mu_{C} C_{i}-\beta_{C} C_{i}^{2} \\
\frac{\mathrm{d} F_{i}}{\mathrm{~d} t} & =\epsilon_{F} \alpha_{F} F_{i} D O M_{i}-\alpha_{R} R_{i} F_{i}-\mu_{F} F_{i}-\beta_{F} F_{i}^{2} \\
\frac{\mathrm{d} R_{i}}{\mathrm{~d} t} & =\epsilon_{R} \alpha_{R} R_{i}\left(G_{i}+S_{i}+C_{i}+F_{i}\right)-\mu_{R} R_{i}-\beta_{R} R_{i}^{2}
\end{aligned}
$$




$$
\begin{aligned}
& \frac{\mathrm{d} N_{i}}{\mathrm{~d} t}=\frac{\delta_{N}}{V_{i}}\left(\sum_{j=1}^{n} w_{j i} N_{j} Q_{j}-N_{i} Q_{i}\right)+\frac{\phi_{N, i}}{V_{i}}-\alpha_{P} l_{i} P_{i} N_{i}-\lambda_{N} N_{i} ; \\
& \frac{\mathrm{dCPOM}}{\mathrm{d} t}=\frac{\delta_{\mathrm{CPOM}}}{V_{i}}\left(\sum_{j=1}^{n} w_{j i} C P O M_{j} Q_{j}-\mathrm{CPOM}_{i} Q_{i}\right)+\frac{\phi_{C P O M, i}}{V_{i}}-\alpha_{S} S_{i} C P O M_{i}-\lambda_{C P O M} C P O M_{i} ;
\end{aligned}
$$

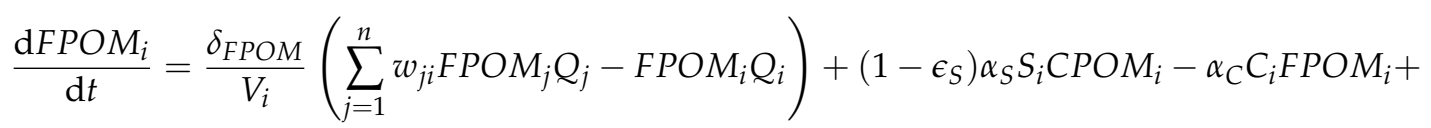

$$
\begin{aligned}
& -\lambda_{F P O M} F_{P O M} \text {; } \\
& \frac{\mathrm{d} D O M_{i}}{\mathrm{~d} t}=\frac{\delta_{D O M}}{V_{i}}\left(\sum_{j=1}^{n} w_{j i} D O M_{j} Q_{j}-D O M_{i} Q_{i}\right)+\frac{\phi_{D O M, i}}{V_{i}}-\alpha_{F} F_{i} D O M_{i}-\lambda_{D O M} D O M_{i},
\end{aligned}
$$

where subscript $i$ identifies a river reach. For the living compartments $X=\{P, G, S, C, F, R\}$ (Eqs. $(1 \mathrm{a}-\mathrm{f})$ ), the rate of change in biomass density $X$ depends on biomass gain owing to feeding (assuming direct dependence of feeding rate on biomass production) and biomass loss due to metabolism, predation and intra-group competition for resources (density dependence). The parameters related to the living compartments $X$ are feeding rate $\alpha_{X}$, efficiency of resource assimilation $\epsilon_{X}$, strength of intra-group competition $\beta_{X}$, and mortality rate $\mu_{X}$. To include the effect of light availability on nutrient uptake of producers, we expressed the realized feeding rate of producers as $\alpha_{P} l_{i}$, where $l_{i}$ is a site-specific light limitation factor. Detailed information on parameter values is available in Table S1.

For the resource compartments $Y=\{N, C P O M, F P O M, D O M\}$ (Eqs. (1g-j)), the rate of change of resource density $Y$ depends on mass gain from local terrestrial inputs and inputs from upstream reaches via hydrological transport; and mass loss due to consumption by living organisms, downstream hydrological transport, and resource degradation and deposition. The parameters related to the abiotic resources $Y$ are the mass of local terrestrial inputs $\phi_{Y, i}$, the relative downstream velocity of resource $Y$ with respect to water $\delta_{Y}$, and the rate of resource loss due to processes other than consumption $\lambda_{Y}$ (i.e., degradation or deposition). $Q_{i}$ and $V_{i}$ represent the water discharge and the water volume of reach $i$, respectively; $w_{j i}$ is the generic entry of the adjacency matrix ( $w_{j i}=1$ if reach $j$ drains into $i$ and 0 otherwise). Following the RCC [Vannote et al., 1980], we assumed that FPOM is a by-product of CPOM consumption by shredders (Fig. 1), therefore inputs of FPOM originate from the aquatic environment only. Importantly, Eqs. (1g-j) outline how the physical attributes of river networks, that is water discharge $Q_{i}$ and volume $V_{i}$, influence the density of resources available locally for the functional groups. In particular, both water discharge and volume determine the velocity at which resources are transported across a given reach, while water volume also influences the concentration of local terrestrial inputs. 


\section{Resource spatial dynamics in dendritic river networks}

We generated a large, virtual river network (so-called optimal channel network, OCN) with the R-package OCNet [Carraro et al., 2020, 2021] in order to study changes in the density of functional groups along a gradient of physical conditions specific to river networks. OCNs are structures that reproduce the topological connectivity and scaling features of real river networks [Rinaldo et al., 2014] and are well suited to study riverine ecological processes [Carraro et al., 2020]. We built the OCN (Fig. 2a) on a square lattice spanning an area of $5625 \mathrm{~km}^{2}$ partitioned into 3688 reaches (see details in Supporting Information).

We derived water discharge $Q_{i}$ and water volume $V_{i}$ (Fig. 2c) across all nodes of the network based on drainage area values (Fig. 2b) via the scaling relationships of Leopold and Maddock [1953]. As a universal geomorphological feature, drainage area is the master variable controlling the physical and hydrological characteristics of a given reach [Leopold et al., 1964; Rodriguez-Iturbe and Rinaldo, 2001], which also warrants its use as a descriptor of the positioning of a reach within the river network [Altermatt, 2013].

We made several assumptions regarding the rate of hydrological transport of resources in order to incorporate the core assumptions of the RCC into our meta-ecosystem model. In particular, we assumed $C P O M$ to be transported downstream at a low rate $\left(\delta_{C P O M}=0.01\right)$ compared to water because of the large size of its constituents, which likely induces clogging [Vannote et al., 1980; Wallace and Webster, 1996]. Conversely, we assumed FPOM to travel downstream at an intermediate rate $\left(\delta_{F P O M}=0.5\right)$, while DOM and $N$ are transported at a high rate (i.e., same velocity as water: $\delta_{D O M}=1$ and $\delta_{N}=1$ ) [Cushing et al., 1993; Wallace and Webster, 1996].

We determined the equilibrium densities of each compartment of the meta-ecosystem in all nodes of the river network by finding a feasible equilibrium state (i.e., non-negative densities at all nodes) for system Eq. (1) by using a linearization method (see Supporting Information for details). We investigated how the densities of functional groups change along a gradient of drainage area and compared the model predictions with the empirical patterns described in the River Continuum Concept [Vannote et al., 1980]. We then compared the spatial distributions and regional biomass of each functional group for two alternative scenarios of resource spatial dynamics: one scenario where hydrological transport of resources is neglected and another scenario where all resources are transported at a high rate (i.e., same velocity as water). All the scenarios were based on the same set of parameters specified in Table S1. 


\section{RESULTS}

The reconstructed physical structure of real-world river networks (Fig. 2a) exhibits predictable distributions of hydrological variables (drainage area, water volume - see Fig. 2b,c), as well as predictable gradients of resource concentration (Fig. 2d) and light availability (Fig. 2e). The input concentration of all resources decreases as drainage area increases (Fig. 2d) because of the corresponding increase in water volume along the downstream direction (Fig. 2c). The change in CPOM input concentration along a gradient of drainage area follows a slightly different pattern than the other resources; this is due to the different mechanism controlling the terrestrial input of such resource with respect to DOM and N (see Supporting Information for a detailed explanation). Light availability (expressed via the light factor $l_{i}$ ) peaks at intermediate values of drainage area (Fig. 2e), while it is lower both in upstream reaches (where the canopy cover provides more shading to the water surface) and in downstream, deeper sites (as light penetration is hampered in high water columns).

The broad-scale relationships between the concentration of terrestrial inputs and drainage area hold true over multiple orders of magnitude in drainage area, and directly affect the functional composition of stream communities in local ecosystems. Our model produces distributions of functional groups across the river network that are in strong agreement with the predictions of the River Continuum Concept (Fig. 3). Specifically, the density of grazers peaks at intermediate values of drainage area (Fig. 3a,f), which is mirrored by the analogous pattern for primary producers (Fig. 3g). Both of these patterns essentially follow the spatial distribution of light availability (Fig. 2e). The density of shredders is maximum in headwaters and decreases as drainage area increases (Fig. 3b,f), while filterers tend to be more homogeneously distributed across the river network (Fig. 3d,f). Conversely, the density of collectors monotonically increases with drainage area (Fig. 3c,f). In 468 nodes (12.7\% of all nodes), all located at the headwaters, collectors are predicted to go extinct. Finally, the density of predators (Fig. 3e), which is proportional to the sum of their preys' densities (Fig. 3f), is relatively constant along a gradient of drainage area. The corresponding spatial patterns of resource densities predicted by the model are shown in Fig. S1.

We then investigate how the rates of hydrological resource flows modify the spatial distribution and regional biomass of the functional groups. We focus on two alternative scenarios, namely a "no flow" scenario (i.e., where hydrological transport of resources is neglected) and a scenario with increased rates of hydrological transport of CPOM and FPOM. In the "no flow" scenario, the absence of hydrological transport of resources has a major effect on the spatial distribution of all functional groups (Fig. 4); indeed, 
in this scenario, the densities of all groups decrease in the downstream direction. Organisms living in headwaters have higher resource availability as no resources flow downstream, while organisms living in downstream reaches face lower resource inputs as they can only rely on local terrestrial inputs. This effect is particularly strong for grazers, filterers and producers (Fig. 4c,e,f), while shredders are the least impacted. Hence, functional groups feeding on fast-flowing resources (i.e., nutrients and $D O M$ ) are more impacted than the ones feeding on slow-flowing resources (i.e., $C P O M$ ) in this scenario.

For the parameter set chosen, the regional biomasses of grazers, producers and filterers are $4.8 \%, 6.7 \%$ and $89.1 \%$ higher in the "no flow" scenario, respectively. For these groups, the increase in density observed in upstream reaches exceeds the decrease observed downstream. Note that this result is sensitive to the type of functional response used in the model (see Supporting Information for additional analyses with a type II functional response). Conversely, the regional biomass of shredders is $9.4 \%$ lower, suggesting that the decrease in CPOM in the most downstream reaches has a significant effect on the regional biomass of this group. The group of collectors exhibit a totally different spatial distribution in the "no flow" scenario, with very high densities in headwaters and a decreasing density in the downstream direction (Fig. 4d). The regional biomass of collectors is more than doubled, which mirrors the amount of FPOM available in this scenario (Fig. S2). As a result, the spatial distribution of predator density changes as well (Fig. 4a), with higher densities in upstream reaches and a regional biomass that is $54.5 \%$ higher without hydrological transport of resources.

In the second scenario, increasing the rates of hydrological transport of CPOM and FPOM has major effects on the spatial distributions and overall quantity of resources available for shredders and collectors at the regional scale (Fig. 5). The amounts of CPOM and FPOM available in the whole river network are $90.8 \%$ and $99.9 \%$ lower in this scenario, respectively (Fig. $5 \mathrm{~d}, \mathrm{e}$ ). These changes have dramatic negative effects on the density of shredders, which is $93.6 \%$ lower at the regional scale, but shows an increasing trend in the downstream direction (Fig. 5b). We also find that shredders go extinct in $28.7 \%$ of the reaches (1058 nodes). Because FPOM is a by-product of shredders activity, the sharp decrease in shredder density cascades to collectors, which go extinct in all reaches in this scenario (Fig. 5c). Consequently, the regional biomass of predators shrinks by more than half (Fig. 5a), with a spatial distribution following those of grazers and filterers, which are unaffected by changes in the rates of hydrological transport of CPOM and FPOM. 


\section{DISCUSSION}

Our work reveals the broad-scale effects of resource spatial dynamics on the structure and functioning of meta-ecosystems in general, and river networks in particular. Specifically, we show that spatially explicit meta-ecosystem models allow understanding the interactive effects of landscape structure and resource spatial dynamics on community functional composition. By performing the first formal integration of the verbal arguments of the River Continuum Concept - a milestone that has been shaping the scientific thinking in freshwater ecology over the last four decades [Doretto et al., 2020; Vannote et al., 1980] - within a meta-ecosystem model, we show that spatial distributions and regional biomasses of major functional groups observed in stream communities are determined by specific rates of resource flows.

Our work corroborates the observation that the general spatial variations of community functional composition described in the River Continuum Concept are driven by the physical attributes of the river network (in particular drainage area), which constrain the dynamics and spatial distribution of resources. Notably, we show that model predictions do not reproduce the spatial distribution of functional groups observed in stream communities when only local ecosystem dynamics are implemented (i.e., the "no flow" scenario), which highlights the central role exerted by hydrological transport of resources in riverine ecosystems. Furthermore, the spatial distributions of functional feeding groups as postulated in the RCC were obtained without making specific assumptions on the spatial distribution of terrestrial resources (e.g., forests being more abundant at higher or intermediate elevation). Hence, not only does our work confirm the verbal arguments of the RCC, but it also demonstrates that drainage area is a powerful metric to assess the vulnerability of specific functional groups of macroinvertebrates to changes in resource flow rates.

Our work illustrates how the formal adaptation of meta-ecosystem theory to real-world landscapes, in this case river networks, allows making quantitative predictions on the local and regional effects of meta-ecosystem dynamics in systems with specific spatial structures. Here, we demonstrated that increased rates of resource flows in river networks have a negative effect on regional biomasses of macroinvertebrates, and strongly modify their spatial distributions. We show how the spatial distribution of functional groups crucially depends on the ratio between the feeding rate of each group and the rate at which their resources are transported across the river network. In particular, shredders are adapted to feed on CPOM, which is a slow-flowing resource. During their feeding activity, shredders produce FPOM, which is the resource on which collectors feed. We show that when the transport of $C P O M$ is accelerated, which could be for instance due to the elimination of low-current areas by human activities, populations of shredders are 
heavily depleted and this, in turn, leads to the extinction of collectors across the whole river network via a massive decrease in FPOM concentration. These results also support the use of metacommunity and meta-ecosystem theory for ecosystem management and restoration, as suggested by Harvey et al. [2017b], by outlining the negative effect of high rates of resource transportation in river networks on several functional groups of macroinvertebrates. The meta-ecosystem approach also formalizes the relationship between the size of the particles constituting a given resource and the rate at which it is transported via hydrological flow. This relationship is based on general physical laws and could be applied to other types of resources and other freshwater organisms, such as fishes or microbes.

Recent studies outlined the need for meta-ecosystem ecology to move from a very simplified and abstract representation of ecosystems to a more realistic one [Gounand et al., 2018a; Guichard, 2019]. Previous theoretical studies on meta-ecosystems demonstrated that high rates of resource flow destabilize simple producer-consumer dynamics [Marleau et al., 2014]. However, most studies on meta-ecosystem dynamics have hitherto focused on the effect of recycling or organism movement on ecosystem stability and productivity in small spatial networks. Here, we show that incorporating a realistic landscape and empirical knowledge of resource spatial dynamics can bring new insights on the effect of meta-ecosystem dynamics on the structure and functioning of real-world ecosystems. By upscaling the meta-ecosystem concept to a large spatial network, we identified broad-scale effects of resource spatial dynamics on the functional composition of stream communities.

Gravel et al. [2010a] defined a meta-ecosystem effect as the effect of the ecosystem composition, structure, and productivity in one locality on the ecosystem dynamics in another locality resulting from spatial flows and recycling of matter. Here, we demonstrate the existence of a strong meta-ecosystem effect in river networks by manipulating the rates of hydrological transport of resources. While we applied our model to river ecosystems, the general approach proposed can be adapted to other ecological systems where meta-ecosystems dynamics are important processes, such as marine shorelines, coral reef systems or estuaries [Gounand et al., 2018a; Menge et al., 2015; Spiecker et al., 2016]. The application of meta-ecosystem theory to real-world landscapes crucially depends on an informed knowledge of the specific physical attributes of a landscape that constrain the direction and rates of resource flows among localities [Polis et al., 2004]. Hence, a systematic quantification of cross-ecosystem flows of resources (e.g. Gounand et al. [2018b]) is central for fostering future developments of spatially explicit meta-ecosystem models and predicting the large-scale effects of human-induced changes in meta-ecosystem dynamics on biodiversity and ecosystem functioning. 


\section{Acknowledgements}

Funding is from the Swiss National Science Foundation Grants No PP00P3_179089 and 31003A_173074 and the University of Zurich Research Priority Programme “URPP Global Change and Biodiversity” (to F.A.) and the University of Zurich Forschungskredit, grant no. K-74335-03-01 (to C.J.).

\section{References}

Abelho, M. and Descals, E. (2019). Litter movement pathways across terrestrial-aquatic ecosystem boundaries affect litter colonization and decomposition in streams. Funct. Ecol., 33(9):1785-1797.

Albert, E. M., Fortuna, M. A., Godoy, J. A., and Bascompte, J. (2013). Assessing the robustness of networks of spatial genetic variation. Ecol. Lett., 16:86-93.

Altermatt, F. (2013). Diversity in riverine metacommunities: a network perspective. Aquat. Ecol., 47(3):365377.

Bartels, P., Cucherousset, J., Steger, K., Eklöv, P., Tranvik, L. J., and Hillebrand, H. (2012). Reciprocal subsidies between freshwater and terrestrial ecosystems structure consumer resource dynamics. Ecology, 93(5):1173-1182.

Carrara, F., Altermatt, F., Rodriguez-Iturbe, I., and Rinaldo, A. (2012). Dendritic connectivity controls biodiversity patterns in experimental metacommunities. P. Natl. Acad. Sci. U.S.A, 109(15):5761-5766.

Carraro, L., Altermatt, F., Fronhofer, E. A., Furrer, R., Gounand, I., Rinaldo, A., and Bertuzzo, E. (2021). OCNet: Optimal Channel Networks. R package version 0.5.0.

Carraro, L., Bertuzzo, E., Fronhofer, E. A., Furrer, R., Gounand, I., Rinaldo, A., and Altermatt, F. (2020). Generation and application of river network analogues for use in ecology and evolution. Ecol. Evol., 10(14):7537-7550.

Cushing, C. E., Minshall, G. W., and Newbold, J. D. (1993). Transport dynamics of fine particulate organic matter in two Idaho streams. Limnol. Oceanogr., 38(6):1101-1115.

Doretto, A., Piano, E., and Larson, C. E. (2020). The river continuum concept: lessons from the past and perspectives for the future. Can J. Fish Aquat. Sci, 77(11):1853-1864. 
Gounand, I., Harvey, E., Little, C. J., and Altermatt, F. (2018a). Meta-Ecosystems 2.0: Rooting the Theory into the Field. Trends Ecol. Evol., 33(1):36-46.

Gounand, I., Little, C. J., Harvey, E., and Altermatt, F. (2018b). Cross-ecosystem carbon flows connecting ecosystems worldwide. Nat. Commun., 9(1).

Gravel, D., Guichard, F., Loreau, M., and Mouquet, N. (2010a). Source and sink dynamics in metaecosystems. Ecology, 91(7):2172-2184.

Gravel, D., Massol, F., and Leibold, M. A. (2016). Stability and complexity in model meta-ecosystems. Nat. Commun., 7(10):12457.

Gravel, D., Mouquet, N., Loreau, M., and Guichard, F. (2010b). Patch dynamics, persistence, and species coexistence in metaecosystems. Am Nat., 176(3):289-302.

Guichard, F. (2019). Recent advances in metacommunities and meta-ecosystem theories. F1000Research, $8: 1279$.

Hanski, I. and Ovaskainen, O. (2000). The metapopulation capacity of a fragmented landscape. Nature, 404(6779):755-758.

Harvey, E., Gounand, I., Fronhofer, E. A., and Altermatt, F. (2020). Metaecosystem dynamics drive community composition in experimental, multi-layered spatial networks. Oikos, 129(3):402-412.

Harvey, E., Gounand, I., Little, C. J., Fronhofer, E. A., and Altermatt, F. (2017a). Upstream trophic structure modulates downstream community dynamics via resource subsidies. Ecol. Evol., 7(15):5724-5731.

Harvey, E., Gounand, I., Ward, C. L., and Altermatt, F. (2017b). Bridging ecology and conservation: from ecological networks to ecosystem function. J. Appl. Ecol., 54(2):371-379.

Larsen, S., Bruno, M. C., Vaughan, I. P., and Zolezzi, G. (2019). Testing the River Continuum Concept with geostatistical stream-network models. Ecol. Complex., 39(March):100773.

Leopold, L. B. and Maddock, T. (1953). The hydraulic geometry of stream channels and some physiographic implications. Technical Report Geological Survey Professional Paper 252, Washington DC.

Leopold, L. B., Wolman, M. G., and Miller, J. P. (1964). Fluvial Processes in Geomorphology. Dover Publications Inc., New York, US. 
Leroux, S. J. and Loreau, M. (2008). Subsidy hypothesis and strength of trophic cascades across ecosystems. Ecol. Lett., 11(11):1147-56.

Leroux, S. J. and Loreau, M. (2012). Dynamics of Reciprocal Pulsed Subsidies in Local and Meta-Ecosystems. Ecosystems, 15(1):48-59.

Loreau, M. and Holt, R. D. (2004). Spatial flows and the regulation of ecosystems. Am Nat., 163(4):606-615.

Loreau, M., Mouquet, N., and Holt, R. D. (2003). Meta-ecosystems: A theoretical framework for a spatial ecosystem ecology. Ecol. Lett., 6(8):673-679.

Marleau, J. N., Guichard, F., and Loreau, M. (2014). Meta-ecosystem dynamics and functioning on finite spatial networks. P. Roy. Soc. B-Biol. Sci., 281(1777):20132094.

Marleau, J. N., Guichard, F., Mallard, F., and Loreau, M. (2010). Nutrient flows between ecosystems can destabilize simple food chains. J. Theor. Biol., 266(1):162-174.

Massol, F., Gravel, D., Mouquet, N., Cadotte, M. W., Fukami, T., and Leibold, M. a. (2011). Linking community and ecosystem dynamics through spatial ecology. Ecol. Lett., 14(3):313-23.

Menge, B. A., Gouhier, T. C., Hacker, S. D., Chan, F., and Nielsen, K. J. (2015). Are meta-ecosystems organized hierarchically? A model and test in rocky intertidal habitats. Ecol. Monogr., 85(2):213-233.

Montagano, L., Leroux, S. J., Giroux, M., and Lecomte, N. (2018). The strength of ecological subsidies across ecosystems: a latitudinal gradient of direct and indirect impacts on food webs. Ecol. Lett., 22(2):ele.13185.

Polis, G. A., Anderson, W. B., and Holt, R. D. (1997). Toward an integration of landscape and food web ecology: the dynamics of spatially subsidized food webs. Annu. Rev. Ecol. S., 28(1):289-316.

Polis, G. A., Power, M. E., and Huxel, G. R. (2004). Food webs at the landscape level. University of Chicago Press.

Rinaldo, A., Rigon, R., Banavar, J. R., Maritan, A., and Rodriguez-Iturbe, I. (2014). Evolution and selection of river networks: Statics, dynamics, and complexity. P. Natl. Acad. Sci. U.S.A, 111(7):2417-2424.

Rodriguez-Iturbe, I. and Rinaldo, A. (2001). Fractal River Basins. Chance and self-organization. Cambridge University Press, New York, US.

Saccheri, I., Kuussaari, M., Kankare, M., Vikman, P., Fortelius, W., and Hanski, I. (1998). Inbreeding and extinction in a butterfly metapopulation. Nature, 392(6675):491-494. 
Schmitz, O. J., Wilmers, C. C., Leroux, S. J., Doughty, C. E., Atwood, T. B., Galetti, M., Davies, A. B., and Goetz, S. J. (2018). Animals and the zoogeochemistry of the carbon cycle. Science, 362(6419).

Soininen, J., Bartels, P., Heino, J., Luoto, M., and Hillebrand, H. (2015). Toward more integrated ecosystem research in aquatic and terrestrial environments. BioScience, 65(2):174-182.

Spiecker, B., Gouhier, T. C., and Guichard, F. (2016). Reciprocal feedbacks between spatial subsidies and reserve networks in coral reef meta-ecosystems. Ecol. Appl., 26(1):264-278.

Vannote, R. L., Minshall, G. W., Cummins, K. W., Sedell, J. R., and Cushing, C. E. (1980). The river continuum concept. Can J. Fish Aquat. Sci, 37(1):130-137.

Wallace, J. B. and Webster, J. R. (1996). The role of macroinvertebrates in stream ecosystem function. Annual Rev. Entomol., 41(1):115-139. 


\section{Figures}

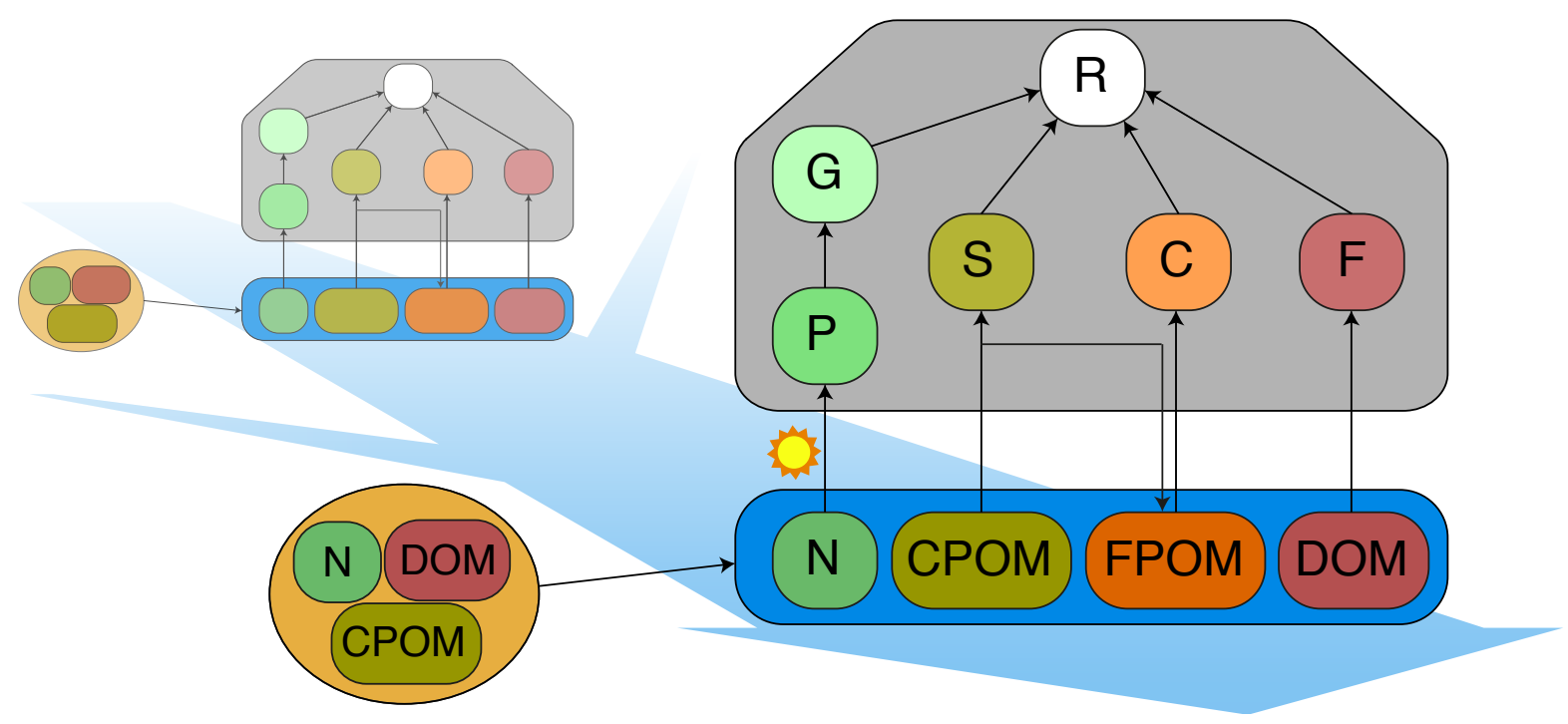

Figure 1: Conceptual illustration of the meta-ecosystem model for river networks. A local stream community (grey box) is composed of a primary producer $P$ and five functional groups of consumers $(G, S, C, F$ and $R$ ). The black arrows illustrate the feeding links between the groups and the abiotic resources (blue box). The resources available for a given local community originate both from local terrestrial inputs (yellow ellipse on the left) and from the ecosystems situated upstream via hydrological transport (blue arrow and transparent boxes). Dissolved nutrients $(N)$ and light are the basal resources for primary producers $P$, while coarse (CPOM), fine (FPOM) and dissolved (DOM) particulate organic matter are the basal resources for shredders $(S)$, collectors $(C)$ and filterers $(F)$, respectively. Predators $(R)$ can feed on all primary consumers, that is $G, S, C$ and $F$. By feeding on $C P O M$, shredders $(S)$ produce FPOM that constitutes the main resource for collectors $(C)$. 

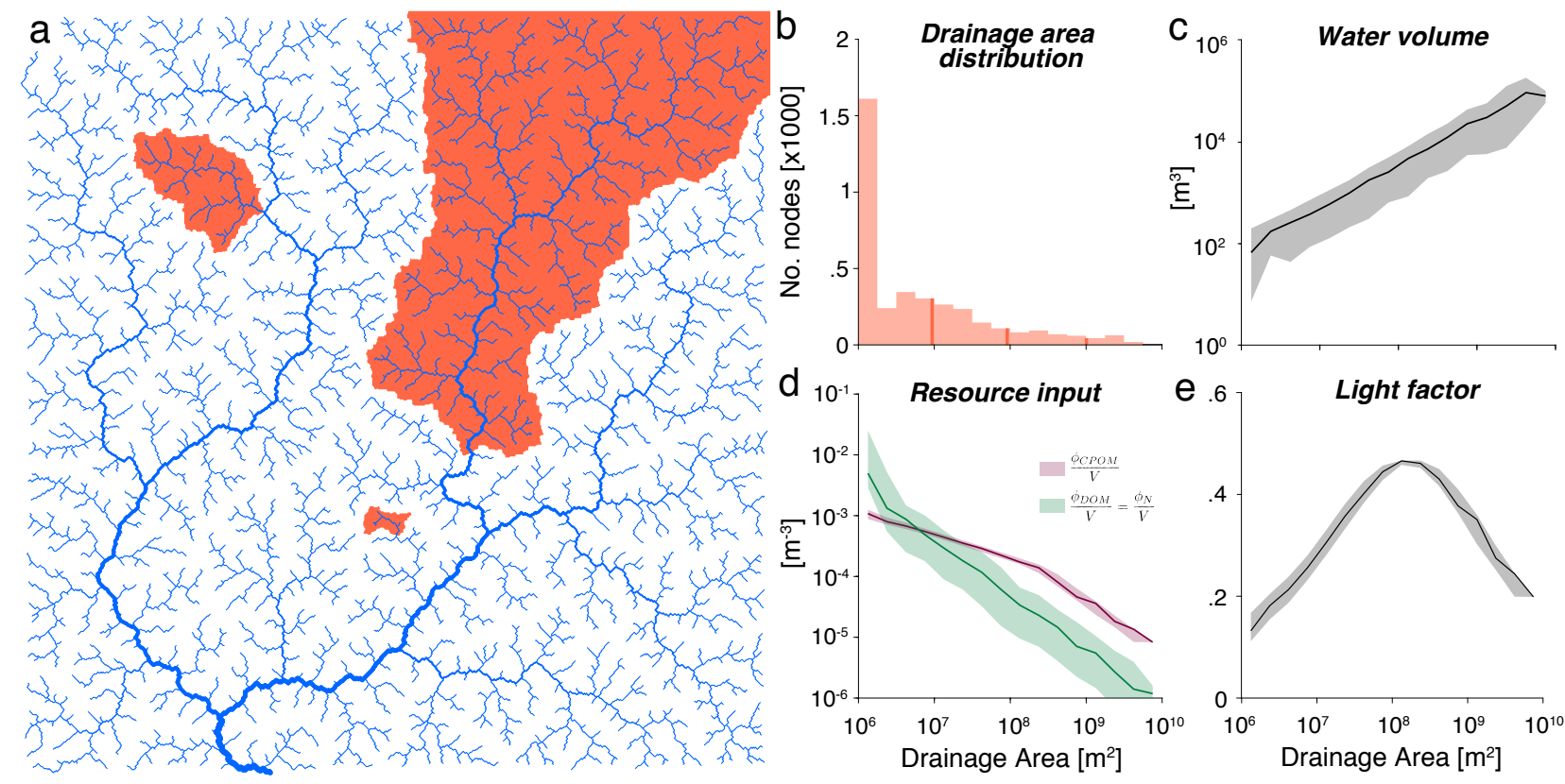

Figure 2: a) The dendritic river network used in the model simulation, spanning a square of area $5625 \mathrm{~km}^{2}$. The river network is partitioned into 3688 nodes (reaches); the drainage areas corresponding to three example reaches are shown in orange. b) Distribution of drainage area values across the 3688 nodes constituting the river network, ranging from 1 to $5625 \mathrm{~km}^{2}$ (grouped into 16 bins). Orange solid lines display the drainage area values corresponding to the three orange areas illustrated in panel a. c-e) Variation of physical attributes of the river network variables along a gradient of drainage area: c) water volume increases with drainage area, d) concentration of terrestrial inputs decreases with drainage area, and e) light availability is maximum for intermediate values of drainage area (around $100 \mathrm{~km}^{2}$ ). Solid lines represent median values for each bin of panel $b$ and shaded areas correspond to the $2.5 \%-97.5 \%$ percentile intervals. 

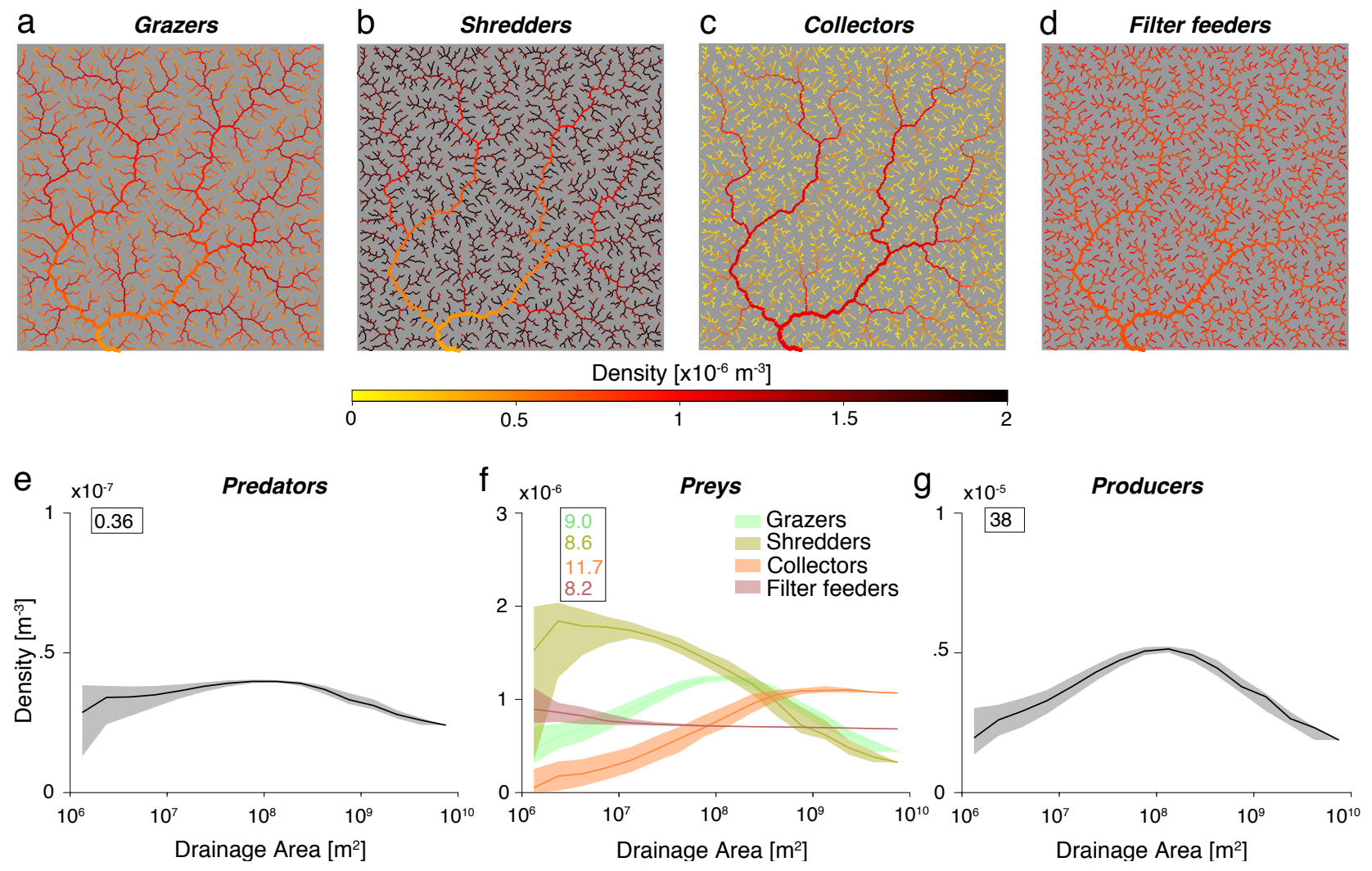

Figure 3: Spatial distributions of functional groups emerging from the meta-ecosystem model. a-d) Map representations of the distributions of the four main functional groups discussed in the RCC. e-g) Variation in density of functional group densities over drainage area. Solid lines represent median values and shaded areas correspond to the $2.5 \%-97.5 \%$ percentile intervals. The numbers within boxes correspond to the regional biomasses of the respective functional groups (total biomass density across the whole river catchment). Note that trends in panel $\mathrm{f}$ correspond to the patterns shown in panels a-d. 
a
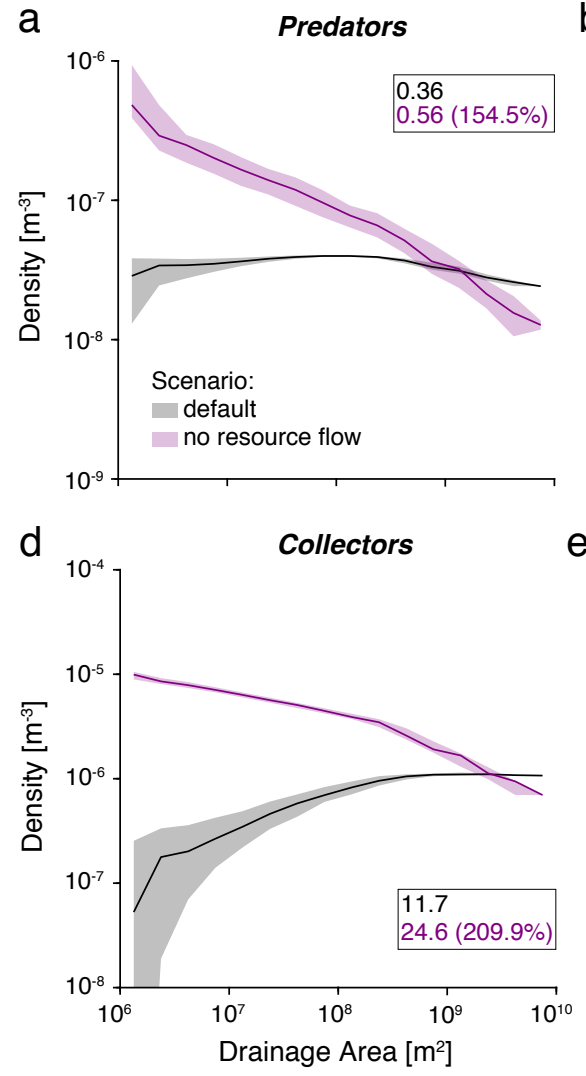

b

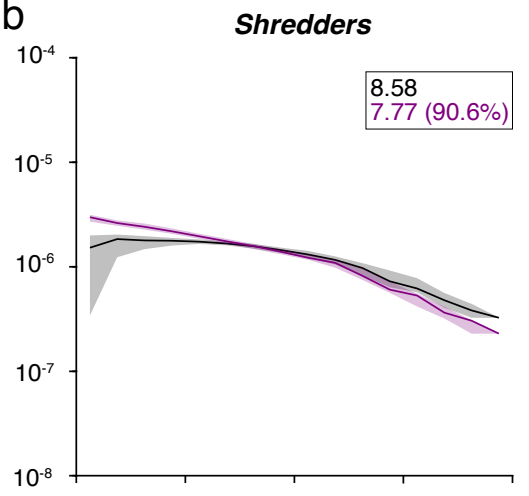

e

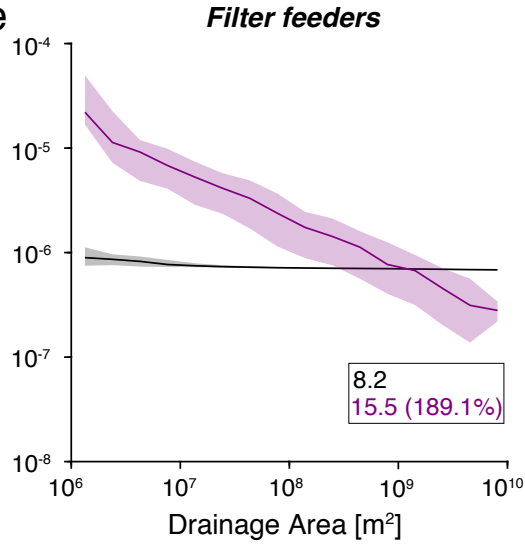

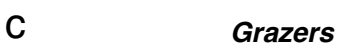
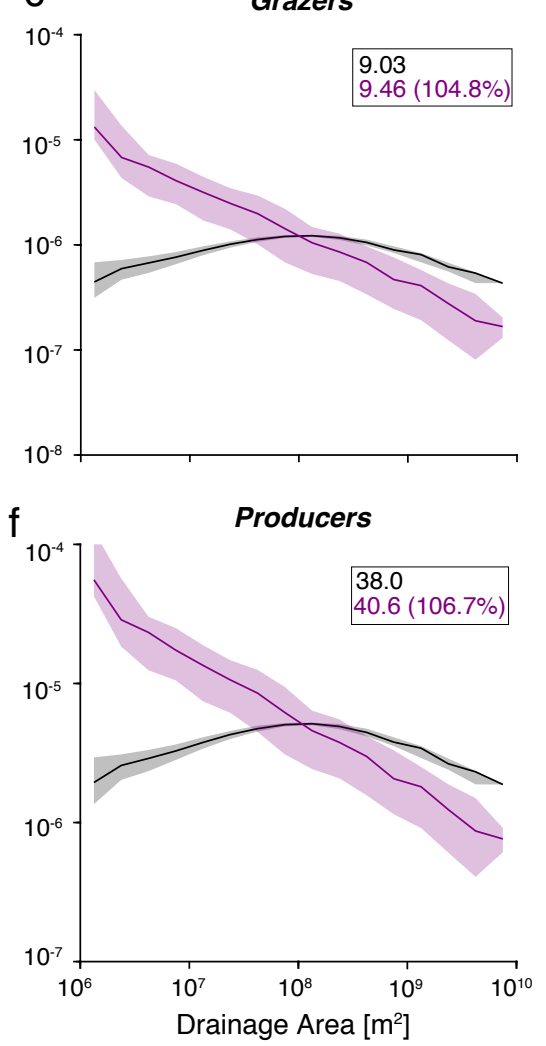

Figure 4: Effect of hydrological transport of resources on the spatial distribution and regional biomass of functional groups in stream communities. Comparison between the spatial distributions of functional groups with (black, same as in Fig. 3) and without (purple) hydrological transport of resources in the river network. Solid lines represent median values and shaded areas correspond to the $2.5 \%-97.5 \%$ percentile intervals. The numbers within boxes correspond to the regional biomasses of the respective groups; percentage values within the boxes indicate the variation in total biomass for the relative resource as compared with the default simulation. The corresponding resource patterns are shown in Fig. S2. 

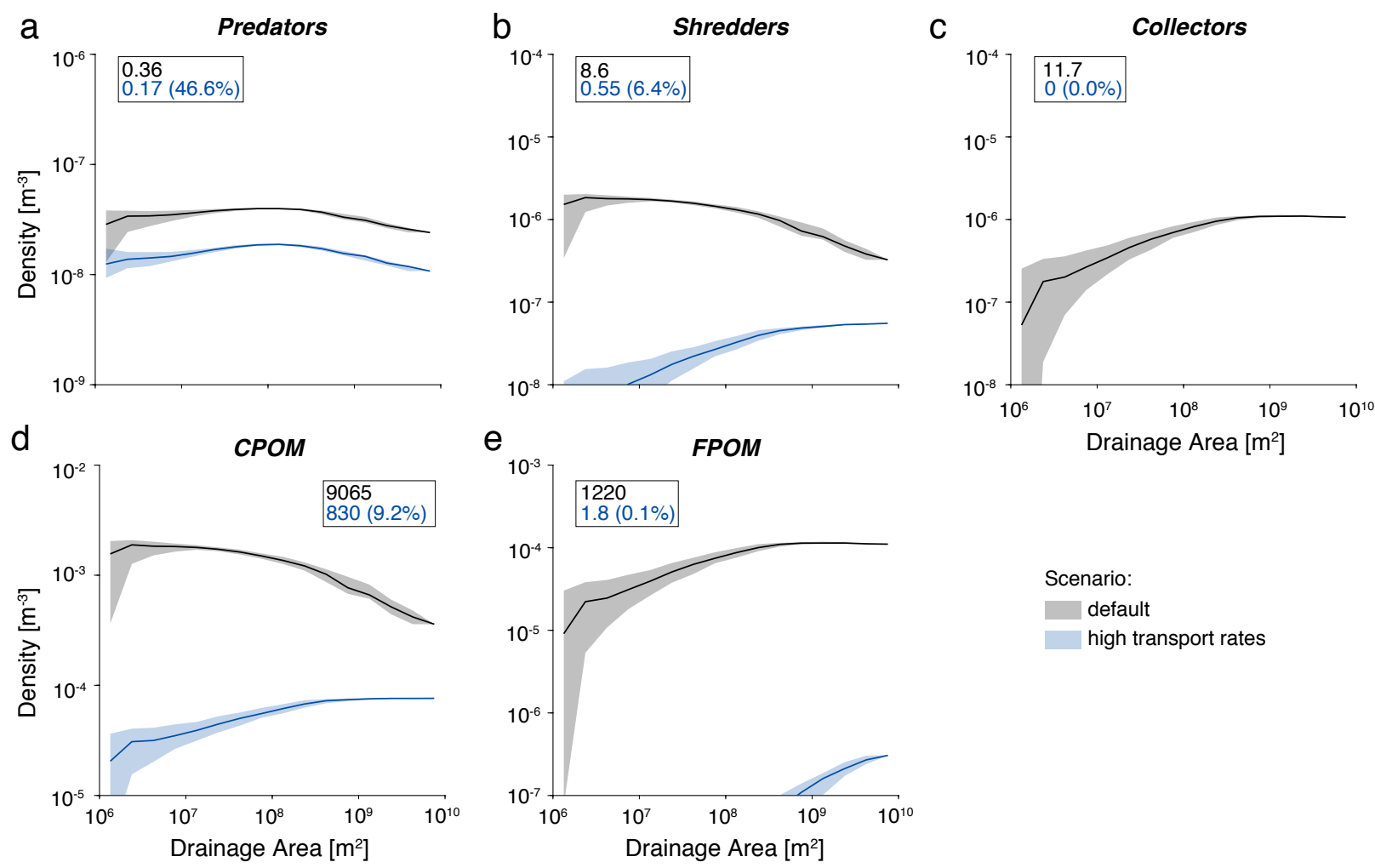

Figure 5: Effect of high rates of hydrological transport of resources on the spatial distribution and regional biomass of functional groups. Comparison between the spatial distributions of functional groups for the default scenario (black, same as in Fig. 3), and the scenario with high rates of hydrological transport for all resources (blue). Solid lines represent median values and shaded areas correspond to the $2.5 \%-97.5 \%$ percentile intervals. The numbers within boxes correspond to the regional biomass of the respective groups; percentage values within the boxes indicate the variation in total biomass for the relative resource as compared with the default simulation. 